Faculty of Science

Faculty Publications

Adsorption of heptane-toluene binary mixtures on a hydrophobic polymer surface Margo Ramsay, Clémentine Beutier, G. Bryce McGarvey, and Dennis K. Hore January 2019

(C) 2019 Ramsay et al.

This article may be downloaded for personal use only. Any other use requires prior permission of the author and AIP Publishing. This article appeared in The Journal of Chemical Physics and may be found at: https://doi.org/10.1063/1.5066555

Citation for this paper:

Ramsay, M.; Beutier, C.; McGarvey, G. B.; \& Hore, D. K. (2017). Adsorption of heptane-toluene binary mixtures on a hydrophobic polymer surface. The Journal of Chemical Physics, 150(1), 014702. DOI: 10.1063/1.5066555 


\section{Adsorption of heptane-toluene binary mixtures on a hydrophobic polymer surface}

Cite as: J. Chem. Phys. 150, 014702 (2019); https://doi.org/10.1063/1.5066555

Submitted: 15 October 2018 . Accepted: 12 December 2018 . Published Online: 03 January 2019

Margo Ramsay, Clémentine Beutier, G. Bryce McGarvey, and Dennis K. Hore (D)

\section{COLLECTIONS}

Paper published as part of the special topic on Nonlinear Spectroscopy and Interfacial Structure and Dynamics Note: This article is part of the Special Topic “Nonlinear Spectroscopy and Interfacial Structure and Dynamics" in J. Chem. Phys.
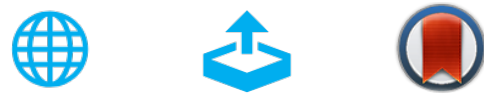

View Online

Export Citation

\section{ARTICLES YOU MAY BE INTERESTED IN}

Water structure at the interface of alcohol monolayers as determined by molecular dynamics simulations and computational vibrational sum-frequency generation spectroscopy

The Journal of Chemical Physics 150, 034701 (2019); https://doi.org/10.1063/1.5072754

Development of ultrafast broadband electronic sum frequency generation for charge dynamics at surfaces and interfaces

The Journal of Chemical Physics 150, 024708 (2019); https://doi.org/10.1063/1.5063458

Bulk-or-interface assignment of heterodyne-detected chiral vibrational sum frequency generation signal by its polarization dependence

The Journal of Chemical Physics 149, 244703 (2018); https://doi.org/10.1063/1.5063290

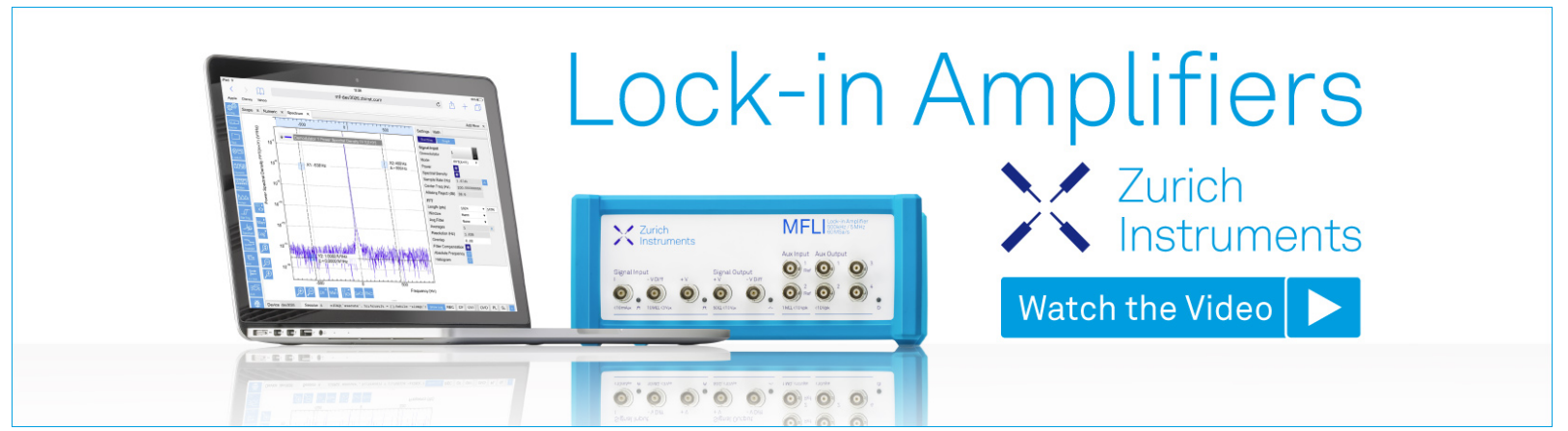




\title{
Adsorption of heptane-toluene binary mixtures on a hydrophobic polymer surface
}

\author{
Cite as: J. Chem. Phys. 150, 014702 (2019); doi: 10.1063/1.5066555 \\ Submitted: 15 October 2018 - Accepted: 12 December 2018 • \\ Published Online: 3 January 2019
}

Margo Ramsay, Clémentine Beutier, ${ }^{\top} \quad$ G. Bryce McGarvey, ${ }^{2}$ and Dennis K. Hore ${ }^{\top}$

\section{AFFILIATIONS}

'Department of Chemistry, University of Victoria, Victoria, British Columbia V8W 3V6, Canada

${ }^{2}$ Sarnia Technology Applications and Research, Imperial Oil, Sarnia, Ontario N7T 8C8, Canada

\begin{abstract}
Polymer coatings offer a means to modulate the adsorption of molecules onto solid surfaces by offering a surface functionality, charge, roughness, and hydrophobicity that is different from the underlying substrate. One application is to provide anti-fouling functions for metal surfaces. Understanding solvent-surface interactions is an essential component to gaining mechanistic insight into the adsorption process. In this work, we study the adsorption of toluene-heptane binary mixtures onto a perflurorinated polymer surface. We use a combination of IR absorption and Raman scattering spectroscopy to study the mixture in the bulk phase, and surface-specific visible-infrared sum-frequency generation to probe the surface layers. Through the use of homoand heterospectral two-dimensional correlation spectroscopy, we conclude that the adsorption of the two solvents is reversible and that the surface structure is generally independent of the surface composition, with a small change in toluene orientation as the toluene content increases. We also find that the hydrophobic fluropolymer has very little preference for either solvent, similar to previous studies on hydrophilic surfaces.
\end{abstract}

Published under license by AIP Publishing. https://oi.org/1 0.1063/1.5066555

\section{INTRODUCTION}

Industrial fouling is the process whereby particles in solution adsorb on surfaces and adversely affect the performance of equipment..$^{1-3}$ This may occur in pipes, reactors, distillation towers, compressors, and heat exchangers. A heat exchanger provides a surface where two materials, usually liquids, can transfer thermal energy. ${ }^{1}$ In the petrochemical industry, heat exchangers are used for the condensation of solvents, heating/cooling of reactors and intermediates, hydrocarbons, and water circuits, and in heat recovery processes. ${ }^{4-6}$ The fouling of heat exchangers alone costs refineries millions of dollars each year as a result of several negative consequences. If the flow is so obstructed that it becomes blocked, this will lead to catastrophic failure. However, even in cases where there is only a relatively small change in the diameter of the conduit, the low thermal conductivity of the adsorbed

Note: This article is part of the Special Topic "Nonlinear Spectroscopy and Interfacial Structure and Dynamics" in J. Chem. Phys. layers compromise the heat exchange, causing some parts of the process to become too hot, while others are at suboptimal temperatures. This in turn leads to undesired pressure gradients, increased energy and maintenance cost, and downtime. The most vulnerable heat exchangers in a refinery are in pre-heat trains for crude oil distillation. ${ }^{7-9}$ Previous studies have revealed that the fouling characteristics are heavily influenced by temperature, flow rate, and the composition of the oil. ${ }^{10}$ Asphaltenes are a class of heavy organic compounds in crude oil that are soluble in toluene, but insoluble in heptane. ${ }^{11,12}$ Although some of the events that trigger asphaltene deposition remain unknown, asphaltenes have been strongly implicated with preheat train fouling, with evidence that they are the major factor controlling the rate of the fouling process. ${ }^{10,13,14}$

Polymer films are currently used in refinery components such as compressors and are being investigated for use in heat exchangers where they can have a significant impact on mitigating fouling. ${ }^{15,16}$ A study on antifouling coatings for petrochemical industries found that multilayer composite coatings with a modified polytetrafluoroethylene (PTFE 
or Teflon) organic topcoat had superior antifouling performance compared with other antifouling coatings. ${ }^{17}$ Rational design attempts to discourage the adsorption of particulates rely on a detailed understanding of the coating surface structure, the coating-liquid interaction, and the coating-adsorbate interaction. While there are techniques capable of monitoring particulate adsorption, there is a paucity of methods that can simultaneously yield meaningful structural data that provide feedback for selection of the coating material. One of the largest obstacles in this characterization is achieving sufficient selectivity for the interfacial species. To understand the adsorption process at the Teflon polymer surface, three types of interactions must be considered: polymer-solution, polymer-adsorbent, and adsorbentsolution. Before transport, heavy oils are blended with other petroleum products to reduce their viscosity and density, and in these blends, asphaltenes rarely exceed $2 \% .^{10}$ Therefore, a thorough understanding of the interactions between the polymer and the solution is crucial since it is the main component in the system.

In this study, we employ a combination of surface-specific vibrational sum-frequency generation (SFG), IR absorption, and Raman scattering spectroscopy to study the interaction of heptane and toluene with a hydrophobic fluoropolymer surface. SFG spectroscopy is an ideal probe of solid-liquid interfaces as it is non-destructive, can be performed in situ, and is specific to surface species on account of the broken centrosymmetry requirement for an even-order optical nonlinearity under the dipole approximation. ${ }^{18-20}$ In an SFG experiment, two intense pulsed lasers with $\omega_{\text {vis }}=12500 \mathrm{~cm}^{-1}$ are overlapped at the buried solid-liquid interface. One of the beams is tuned over the mid-infrared region of interest $\omega_{\mathrm{IR}}$, and the resulting SFG at $\omega_{\mathrm{vis}}+\omega_{\mathrm{IR}}$ is detected. When the SFG signal is displayed as a function of $\omega_{\mathrm{IR}}$, we have the ability to study molecular vibrations in a manner analogous to IR and Raman spectroscopy, but probing only species directly at the interface on account of their orientation preference when interacting with the fluoropolymer film. A previous study has investigated mixtures of toluene with various straight-chain alkanes including heptane on a hydrophilic silica surface; ${ }^{21}$ this is an opportunity for us to contrast this with a hydrophobic surface. We also demonstrate the utility of two-dimensional correlation analysis of the SFG and SFG-bulk vibrational spectra, particularly for cases such as this where the spectral region is highly congested, and fitting the spectra is not robust or practical. 22,23

\section{EXPERIMENTAL}

A 3:1 solution of $\mathrm{H}_{2} \mathrm{SO}_{4}: \mathrm{H}_{2} \mathrm{O}_{2}$ was used to clean a $70^{\circ}$ doveshaped IR-grade fused silica prism (Del Mar Photonics, San Diego, CA), fluoropolymer O-ring (Marco Rubber, Seabrook, $\mathrm{NH}$ ), and custom Teflon sample cell (see Fig. 1) between each set of experiments. All parts were then rinsed and sonicated in $18.2 \mathrm{M} \Omega \mathrm{cm}$ water three times and stored in an air-tight container prior to use. A $2 \%$ by weight solution of Teflon

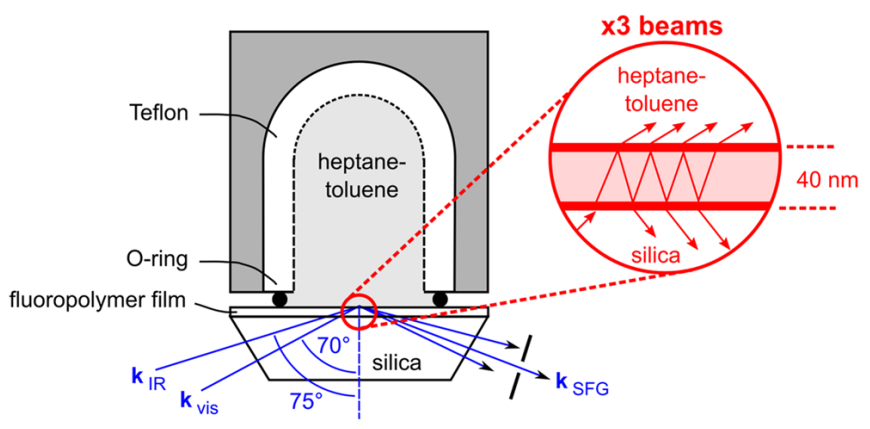

FIG. 1. Visible-infrared sum-frequency generation in a reflection geometry at the polymer-liquid interface. Thin film interference is depicted for a single beam in the inset, although this must be considered for all three beams.

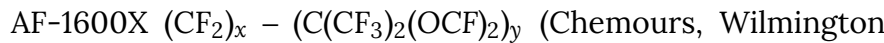
$\mathrm{DE}$ ) in Fluorinert FC-40 (Sigma Aldrich, Oakville, ON) was spin coated onto the large square face of the prism at $3000 \mathrm{rpm}$. The prism with film coating was then annealed at $180^{\circ} \mathrm{C}$ (approximately $20^{\circ} \mathrm{C}$ above the polymer glass transition temperature) for $6 \mathrm{~h}$, and then cooled slowly to room temperature before pressing it against the O-ring to form a liquid-tight seal against the Teflon enclosure. Using separate samples to avoid contamination, a nominal film thickness of $40 \mathrm{~nm}$ was determined with a stylus profilometer (Bruker Dektak XT) by scratching the films with a sharp metal tip, and then scanning in a direction perpendicular to the scratch. HPLC grade heptane (Fischer Chemicals) and toluene (Fischer Chemicals) were used to prepare binary mixtures of the two solvents. We found it critical to avoid buying heptane in bottles with rubber septa, due to trace polydimethylsiloxane contamination that ends up on the surface co-adsorbed with heptane.

Details of our picosecond wavelength-scanning SFG spectrometer have been described previously. ${ }^{24}$ The important details for this work are the fixed-frequency visible beam wavelength of $532 \mathrm{~nm}$ and angle of $70^{\circ}$, and the incident infrared beam angle of $75^{\circ}$. These values are used in subsequent calculations of the optical constants and local field corrections, as will be described in detail below. It was initially challenging to prepare samples that did not produce $\mathrm{SFG}$ in the water $\mathrm{O}-\mathrm{H}$ stretching region. We suspect that, since our experimental system (solvents, substrate film surface, sample holder, and O-ring) was entirely hydrophobic, small cracks or pinhole defects in the Teflon film would reveal the hydrophilic silica surface underneath, and that this would be enough to attract even a minute quantity of water from the environment. This was eventually mitigated by a slightly increased temperature and longer time that the films were annealed after spin-coating, presumably healing such defects.

In a separate set of experiments, bulk binary solvent mixtures were prepared in glass vials and Raman spectra with Stokes shifts in the range $400-3800 \mathrm{~cm}^{-1}$ were collected (Renishaw inVia) using an excitation wavelength of $532 \mathrm{~nm}$. A low numerical aperture ensured that the focus 
of the laser was sufficiently in the bulk of the liquid, so there was effectively no Raman signal from the glass. The same solutions were deposited on a single-bounce diamond attenuated total internal reflection (TIR) IR spectrometer (Perkin-Elmer Spectrum Two) to collect spectra in the range $450-4000 \mathrm{~cm}^{-1}$.

\section{RESULTS AND DISCUSSION}

\section{A. Bulk solution spectra}

\section{IR and Raman spectra}

Unprocessed IR and Raman spectra of pure heptane and pure toluene are shown in Fig. S1. In Fig. 2 we show six IR and Raman spectra including the pure liquids and four binary mixtures prepared with $0.2,0.4,0.6$, and 0.8 weight fraction toluene. Heptane has 63 normal modes; toluene has 39 normal
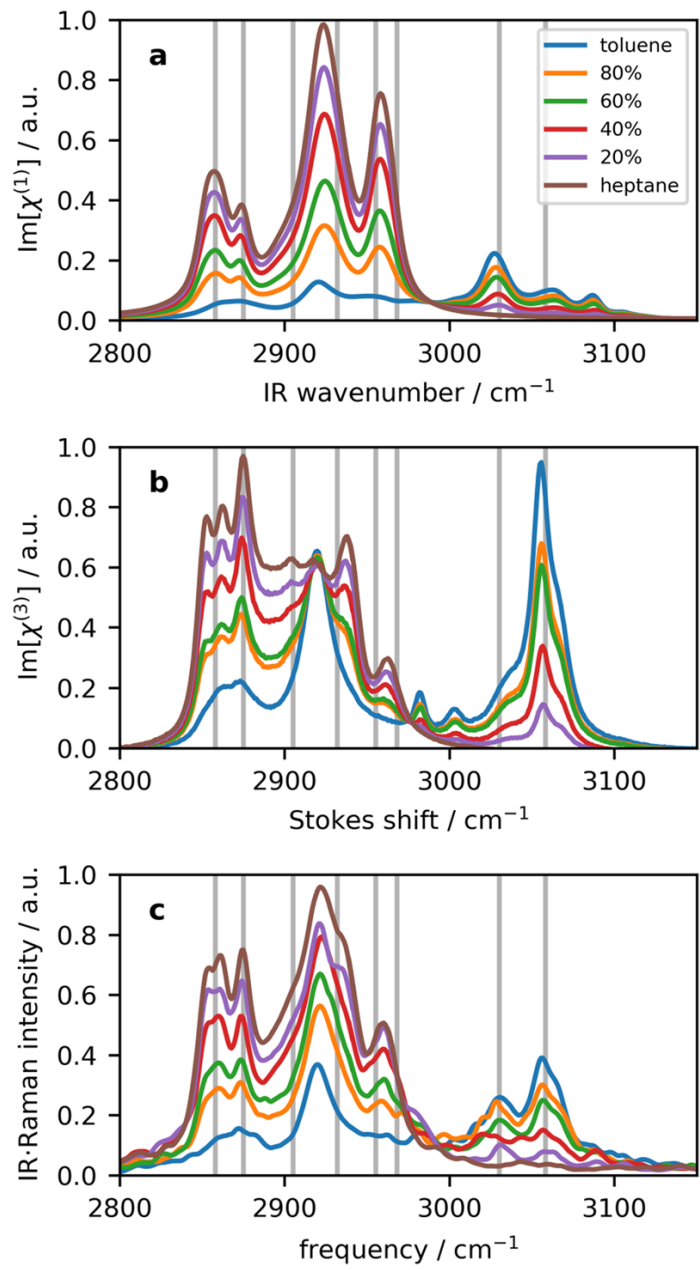

FIG. 2. (a) $\operatorname{Im}\left\{\chi^{(1)}\right\}$ spectra generated from applying the ATR correction to the absorbance spectra, (b) $\operatorname{Im}\left\{\chi^{(3)}\right\}$ spectra generated from the Raman data, and (c) combined IR-Raman spectral intensity, where the weight fraction of toluene is indicated. modes. Of these 102 combined vibrations, 17 have frequencies above $2800 \mathrm{~cm}^{-1}$. 25,26 This is accounting for fundamentals only, without considering additional modes due to combination bands, overtones, or Fermi resonances. Detailed mode assignments for both molecules may be found in the literature. ${ }^{21,27-29}$ The reflection absorbance (RA) was obtained from $R A=-\log _{10}\left(R / R_{0}\right)$, where $R$ is the reflected intensity at the diamond-liquid interface, and $R_{0}$ was measured at the clean diamond-air surface. We draw attention to only a few frequencies of interest as indicated in Fig. 2 (and all other spectra afterwards). They are the eight vibrational modes of interest $\omega_{1}-\omega_{8}$ marked by the vertical grey lines, with an assignment provided in Table I. The lowest frequency mode of interest to us near $\omega_{1}=2858 \mathrm{~cm}^{-1}$ is attributed to the heptane methylene symmetric stretch, followed by the methyl symmetric stretch at $\omega_{2}=2875 \mathrm{~cm}^{-1}$. We draw attention to two other heptane modes, $\omega_{4}=2932 \mathrm{~cm}^{-1}$ and $\omega_{5}=2955 \mathrm{~cm}^{-1}$, assigned to the methylene and methyl antisymmetric stretch, respectively. For toluene, we mark the methyl symmetric and asymmetric stretches at $\omega_{3}=2905 \mathrm{~cm}^{-1}$ and $\omega_{6}=2968 \mathrm{~cm}^{-1}$, respectively, and two aromatic $\mathrm{C}-\mathrm{H}$ stretching modes $\omega_{7}=3030 \mathrm{~cm}^{-1}\left(v_{7 \mathrm{~b}}\right)$, and $\omega_{8}=3058 \mathrm{~cm}^{-1}\left(v_{2}\right)$.

This reflection absorbance was then expressed as the imaginary part of the linear susceptibility $\chi^{(1)}$ using

$$
\operatorname{Im}\left\{\chi^{(1)}\right\}=4 \pi n_{1}^{2} \cos \theta \sqrt{\sin ^{2} \theta-\left(n_{2} / n_{1}\right)^{2}} \cdot \mathrm{RA}
$$

where $n_{1}$ is the refractive index of diamond, constant at 2.38 over this relatively narrow region of the mid-infrared, 30 and $n_{2}$ is the complex refractive index of the solvent mixture. This was estimated using the mole fraction of toluene in the Lorentz-Lorenz effective medium approximation ${ }^{31,32}$ with literature values of the infrared refractive index of both liquids. ${ }^{33}$ Equation (1) simultaneously corrects for the frequency-dependence of the evanescent wave penetration depth above the critical angle, and the shift in frequency that occurs in RA spectra on account of the dispersive contribution from $\mathbb{R e}\left\{n_{2}\right\} .^{34,35}$ The resulting $\operatorname{Im}\left\{\chi^{(1)}\right\}$ spectra are shown in Fig. 2(a).

A similar set of considerations has been used to express the scattered Raman intensity in terms of $\operatorname{Im}\left\{\chi^{(3)}\right\}\left[\right.$ Fig. 2(b)], ${ }^{36}$ this time by dividing by $\omega_{0}\left(\omega_{0}-\omega_{i}\right) \cdot{ }^{37}$ Although this is a very minor correction to the Raman lineshape, it enables us to obtain the combined IR-Raman spectra described below.

TABLE I. Assignments for select vibrational modes of interest.

\begin{tabular}{lclc}
\hline \hline Mode & Freq $\left(\mathrm{cm}^{-1}\right)$ & Molecule & Assignment \\
\hline$\omega_{1}$ & 2858 & Heptane & $\mathrm{CH}_{2} \mathrm{SS}$ \\
$\omega_{2}$ & 2875 & Heptane & $\mathrm{CH}_{3} \mathrm{SS}$ \\
$\omega_{3}$ & 2905 & Toluene & $\mathrm{CH}_{3} \mathrm{SS}$ \\
$\omega_{4}$ & 2932 & Heptane & $\mathrm{CH}_{2} \mathrm{AS}$ \\
$\omega_{5}$ & 2955 & Heptane & $\mathrm{CH}_{3} \mathrm{AS}$ \\
$\omega_{6}$ & 2968 & Toluene & $\mathrm{CH}_{3} \mathrm{AS}$ \\
$\omega_{7}$ & 3030 & Toluene & $\mathrm{CH} v_{7 b}$ \\
$\omega_{8}$ & 3058 & Toluene & $\mathrm{CH} v_{2}$ \\
\hline \hline
\end{tabular}




\section{Composite IR-Raman spectra}

We have recently illustrated the utility of generating a bulk vibrational spectrum where each resonance is described by an oscillator strength that is the product of the IR transition dipole moment and the Raman transition polarizability. ${ }^{38}$ Such spectra, although never realized in any experiment, are particularly useful for comparison with SFG spectra. This is because the vibrational hyperpolarizability may be expressed as the product of these two quantities. However, since the hyperpolarizabity is a tensor of rank three, the isotropic average of its achiral components is zero. The combined IR-Raman spectra on the other hand, as it is derived from bulk quantities, have a non-zero isotropic average and so provide an orientationally-insensitive quantity with the same molecular origins as the SFG signal. In Ref. 38, we describe the detailed procedure for generating these spectra from the experimental $\operatorname{Im}\left\{\chi^{(1)}\right\}$ and $\operatorname{Im}\left\{\chi^{(3)}\right\}$ spectra to produce a spectrum with oscillator strength $\langle\partial \alpha / \partial \mathrm{Q}\rangle\langle\partial \mu / \partial \mathrm{Q}\rangle$, rather than $\langle\partial \alpha / \partial \mathrm{Q} \cdot \partial \mu / \partial \mathrm{Q}\rangle$. Here, $\partial \alpha / \partial \mathrm{Q}$ is the polarizability derivative with respect to the normal mode coordinate, and $\partial \mu / \partial \mathrm{Q}$ is the corresponding dipole moment derivative, both evaluated at the equilibrium geometry. The angle brackets indicate an ensemble average. In brief, our procedure is based on fitting to a continuous distribution of Lorentzians, each narrower than the experimental spectral resolution, to construct the product of the extracted oscillator strengths, followed by a reconstruction of the spectra using the original basis set of Lorentzians. This method is not based on any peak picking and therefore makes no assumptions about the underlying frequency, amplitude, or width of any vibrational modes. The resulting spectra are shown in Fig. 2(c) and, as can be seen, share characteristics common to the $\operatorname{Im}\left\{\chi^{(1)}\right\}$ and $\operatorname{Im}\left\{\chi^{(3)}\right\}$ spectra from which they are derived.

\section{B. SFG spectra}

\section{Intensity correction}

SFG intensity spectra with s-polarized SFG detected, s-polarized visible input, and p-polarized IR input (ssp polarization) for various volume fractions of toluene are shown in Fig. 3(a). The dominant trends are that spectral features decrease with increasing toluene concentration, and it is difficult to observe features above $3000 \mathrm{~cm}^{-1}$. From a closer inspection of the pure toluene spectra (Fig. S1), we know that aromatic $\mathrm{C}-\mathrm{H}$ stretching vibrations are present, just at a lower intensity than the toluene aliphatic $\mathrm{C}-\mathrm{H}$ stretching, and a much lower intensity than any of the heptane vibrations. However, there is a significant difference in the refractive index of the two liquids, and toluene has a higher index than our polymer surface. We therefore transition from a total internal reflection (TIR) regime for heptane, to external reflection for toluene. The intensity in an SFG experiment is proportional to the magnitude squared of the effective second-order susceptibility $\chi^{(2)}$

$$
\mathrm{I}_{\mathrm{SFG}} \propto \frac{\omega_{\mathrm{IR}}^{2} \sec ^{2} \theta_{\mathrm{SFG}}}{n_{\mathrm{SFG}} n_{\mathrm{vis}} n_{\mathrm{IR}}}\left|\chi_{\mathrm{eff}}^{(2)}\right|^{2} \mathrm{I}_{\mathrm{vis}} \mathrm{I}_{\mathrm{IR}}
$$
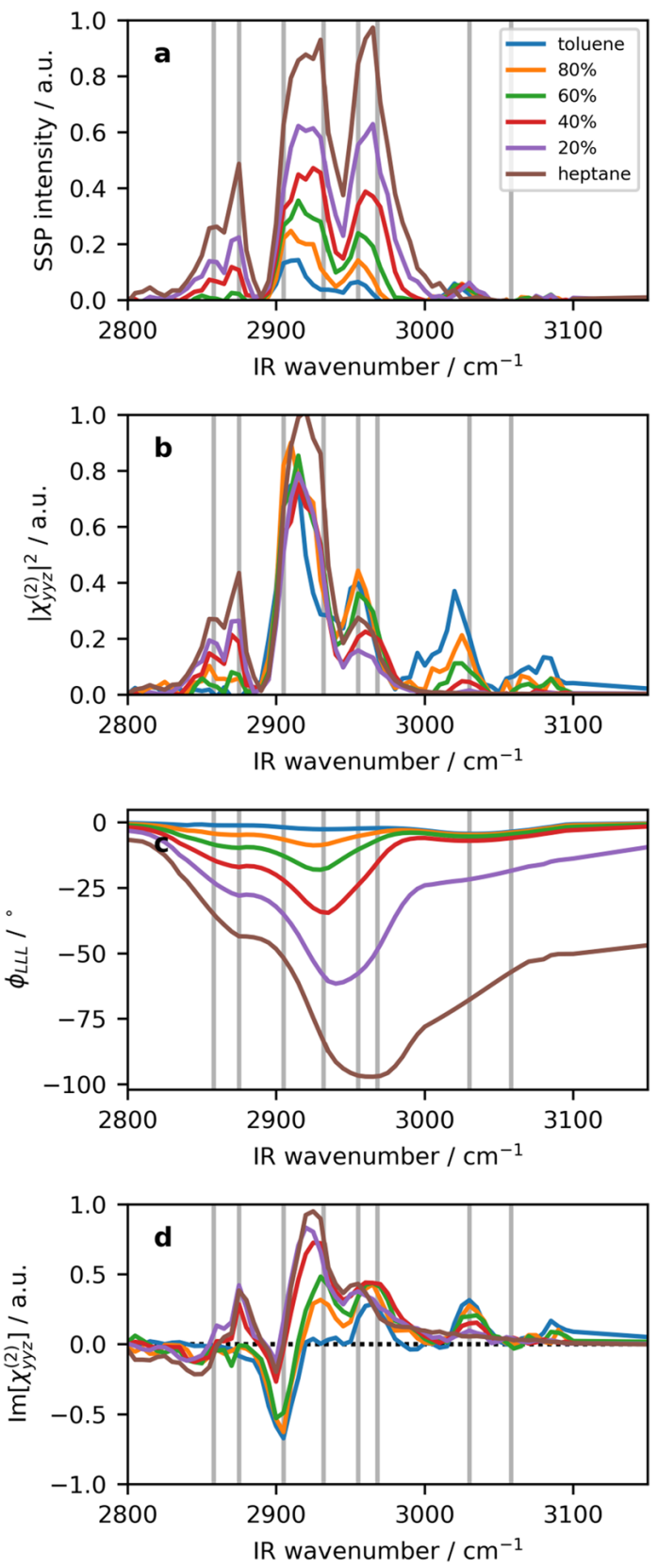

FIG. 3. (a) SSP intensity spectra, (b) local field-corrected spectra to produce $\left|\chi_{y y z}^{(2)}\right|^{2}$, (c) phase of the $L_{y y, S F G} \cdot L_{y y, v i s} \cdot L_{z z, I R}$ contribution, and (d) resulting $\operatorname{Im}\left\{\chi_{y y z}^{(2)}\right\}$ spectra, where the weight fraction of toluene is indicated.

where the refractive indices in the denominator are for the incident phase (polymer thin film), and the reflected angle of the SFG beam $\theta_{\text {SFG }}$ may be obtained from phase-matching considerations knowing the frequencies and angles of the incident visible and IR beams, and the refractive indices of both adjacent phases. The index of the heptane-toluene mixture has already been described above in the determination of 
$\operatorname{Im}\left\{\chi^{(1)}\right\}$. The effective susceptibility is related to the actual surface susceptibility through the local field corrections $\mathbf{L}$. In the ssp polarization scheme, we probe a combination of $y y x$ and $y y z$ elements of $\chi^{(2)}$. As there is no orientation of the polymer in the $(x, y)$ plane of the film, it is not possible for the substrate to impart any orientation on the heptane or toluene molecules that would break their rotational $\mathrm{C}_{\infty v}$ symmetry at the surface. We therefore have $\chi_{y y x}^{(2)}=0$, and the ssp experiments probes a single element of the second-order susceptibility tensor. We can express the relationship between this non-zero yyz element and the effective susceptibility as

$$
\chi_{\mathrm{eff}, \mathrm{ssp}}^{(2)}=\mathrm{L}_{y y, \mathrm{SFG}} \cdot \mathrm{L}_{y y, \mathrm{vis}} \cdot \mathrm{L}_{z z, \mathrm{IR}} \cdot \chi_{y y z}^{(2)}
$$

where the local field correction factors are given by

$$
\mathbf{L}=\left[\begin{array}{ccc}
1-\tilde{r}_{p} & 0 & 0 \\
0 & 1+\tilde{r}_{\mathrm{s}} & \\
0 & 0 & 1+\tilde{r}_{p}
\end{array}\right]
$$

Here, $\tilde{r}_{p}$ and $\tilde{r}_{s}$ are the p- and s-polarized electric field reflection coefficients that are calculated for the three-phase prism-polymer-liquid system. This can be solved using standard algebraic approaches, or via the matrix formulation developed by Abelès, ${ }^{39-41}$ resulting in

$$
\tilde{r}=-\frac{\left(q_{2} q_{3}-q_{1} q_{2}\right) \cos \beta+i\left(q_{1} q_{3}-q_{2}^{2}\right) \sin \beta}{\left(q_{2} q_{3}+q_{1} q_{2}\right) \cos \beta+i\left(q_{1} q_{3}+q_{2}^{2}\right) \sin \beta}
$$

where $\tilde{r}_{p}$ is formed using $q_{i}=\cos \theta_{i} / n_{i}, \tilde{r}_{s}$ using $q_{i}=n_{i} \cos \theta_{i}$, and $\beta=2 \pi d q_{2} / \lambda_{0}$ with $d$ the film thickness and $\lambda_{0}$ the wavelength of each beam in air. Here and in Eq. (5), the subscript 1 refers to the fused silica prism, 2 the polymer film, and 3 the heptane-toluene mixture. Massari and O'Brien have recently demonstrated that such modelling is important when there are multiple sources of SFG signal that are spatially separated, whose interference depends on the distance between the origin of the signals. ${ }^{42,43}$ In our case, there is only one source of SFG, as the fluoropolymer and underlying silica prism do not produce any resonant or non-resonant SFG in the 2800$3200 \mathrm{~cm}^{-1}$ region. Nevertheless, we have recently shown that the same thin-film interference considerations are important, even with a single source of SFG (heptane and/or toluene in this case), as it modulates the surface field amplitude and phase. ${ }^{44}$

An example for the case of pure heptane is shown in Fig. 4. For reference, results that would be obtained for the bare silica prism-heptane interface are indicated with the horizontal dashed black line. These match the wavelength and thickness-dependent three-layer model as the polymer film thickness approaches zero. The other point of interest comes from considering the polymer film to be thick enough so as to ignore interference effects arising from multiple reflections. This solution is indicated in red and provides a substantially different amplitude of the local field corrections. Naturally, the phase of the (bulk) polymer-solution correction term is zero, since the fluoropolymer has a low refractive index (near 1.30) and provides for external refection. Finally
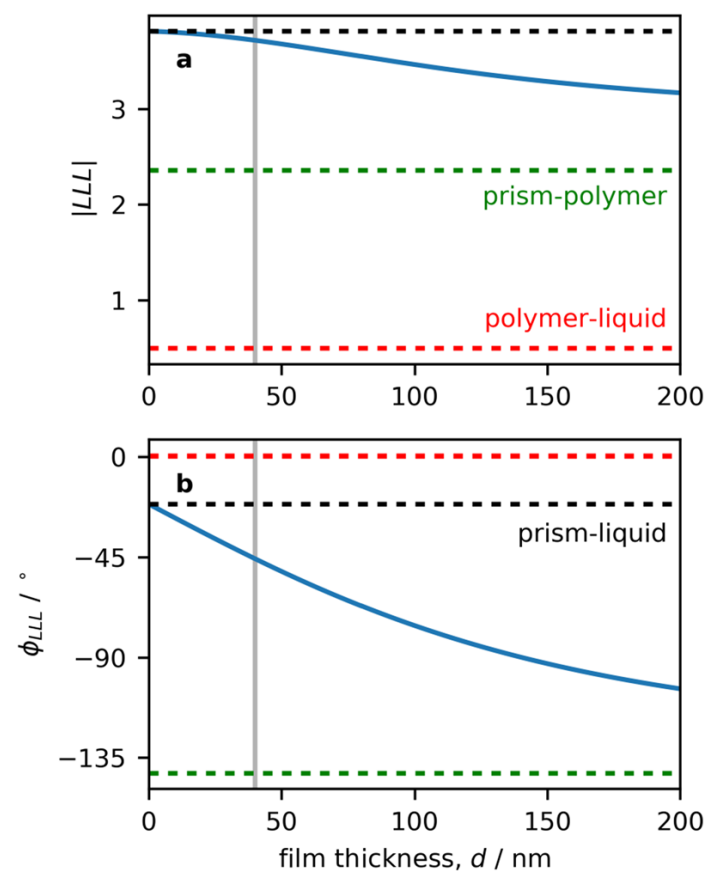

FIG. 4. Effect of thin film interference on the local field correction factors as a function of the film thickness $d$, demonstrated for heptane at $\omega_{\mathrm{IR}}=2875 \mathrm{~cm}^{-1}$. One can see that as $d \rightarrow 0$, the model approaches what would be calculated for the two-phase prism-liquid interface (black line). As $d \rightarrow \infty$, the calculation retrieves the prism-bulk polymer values (green line). It is also important to note that the bulk-polymer-liquid values (red line) are not a good approximation for this system.

we illustrate the limiting case of the silica-polymer interface. This interface is not of interest in the current study, but represents the limit of the thin-film model as the polymer thickness approaches infinity. All of these so-called optical effects can be removed from the $\left|\chi_{\text {eff }}^{(2)}\right|^{2}$ lineshape by calculating $\left|L_{y y, S F G} \cdot L_{y y, v i s} \cdot L_{z z, I R}\right|^{2}$ (hereafter referred to simply as $|L L L|^{2}$ ) and then dividing the intensity by this expression to obtain the spectrum of $\left|\chi_{y y z}^{(2)}\right|^{2}$ shown in Fig. 3(b). Although the uncorrected spectra in Fig. 3(a) show a monotonic decrease in the aliphatic $\mathrm{C}-\mathrm{H}$ stretching modes with decreasing heptane concentration, trends in the aromatic $\mathrm{C}-\mathrm{H}$ stretching region are clear only after the local field corrections (accounting for the change from TIR to external reflection) are applied. In the corrected spectra, there are no longer obvious trends in the aliphatic $\mathrm{C}-\mathrm{H}$ stretching range, but this is not surprising since both heptane and toluene display methyl resonances.

\section{Phase retrieval}

Although these $\left|\chi_{y y z}^{(2)}\right|^{2}$ spectra may be compared with the bulk IR-Raman spectra, there are several advantages to instead obtaining the $\operatorname{Im}\left\{\chi_{y y z}^{(2)}\right\}$ spectra for the discussion. In the case of true $\operatorname{Im}\left\{\chi^{(2)}\right\}$ spectra as would result from a heterodyne SFG experiment in which the phase is explicitly measured, the sign of peaks reveal the absolute direction of 
various chemical functional groups. In our case, however, we will resort to using a maximum entropy method (MEM) to estimate the phase. ${ }^{45-48}$ This technique has been shown to reasonably reproduce the relative $\chi^{(2)}$ phase spectrum; there is a phase offset, the so-called MEM error phase $\phi_{\text {offset }}$, that needs to be determined by external means. ${ }^{45,49,50}$ Since all of the media (fluropolymer, heptane, and toluene) are transparent in the visible region, we can reasonably restrict the MEM error phase to the values that produce $\operatorname{Im}\left\{\chi^{(2)}\right\} \rightarrow 0$ in the region outside of vibrational resonances. In other words, we require any vibrationally non-resonant (purely electronic contribution) to $\chi^{(2)}$ to be real. In practice, this will exist strictly for two values of the MEM error phase that differ by $180^{\circ}$. Before we get to this matter, there is one further detail that needs to be addressed. The constraint of real-valued nonresonant SFG applies strictly to $\chi^{(2)}$, not $\chi_{\text {eff }}^{(2)}$. This is an important consideration for our data, as we work near the critical angle, and have additional phase contributions from the multiple beam interference. ${ }^{44}$ In the case of external reflection, L may be complex-valued on account of the complex-valued refractive indices appearing in $r_{p}$ and $r_{s}$, and again explicitly in $\mathrm{L}_{z z}$. For internal reflection above the critical angle, the phase of LLL

$$
\phi_{\mathrm{LLL}}=\arctan \left[\frac{\operatorname{Im}\left\{\mathrm{L}_{y y, \mathrm{SFG}} \cdot \mathrm{L}_{y y, \mathrm{vis}} \cdot \mathrm{L}_{z z, \mathrm{IR}}\right\}}{\operatorname{Re}\left\{\mathrm{L}_{y y, \mathrm{SFG}} \cdot \mathrm{L}_{y y, \mathrm{vis}} \cdot \mathrm{L}_{z z, \mathrm{IR}}\right\}}\right]
$$

becomes even more significant, as illustrated in Fig. 3(c). Here, we can see that for toluene, there is only a small deviation from $0^{\circ}$; that is, LLL is mostly real. However, in the case of heptane, there are many IR frequencies for which the phase of LLL is close to $90^{\circ}$. When constraining the MEM error phase, we therefore need to take $\phi_{\text {LLL }}$ into consideration. The phase reconstruction is then performed according to

$$
\chi_{y y z}^{(2)}=\left|\chi_{y y z}^{(2)}\right| \exp \left[i\left(\phi_{\mathrm{MEM}}+\phi_{\text {offset }}-\phi_{\mathrm{LLL}}\right)\right]
$$

and the desired imaginary spectra shown in Fig. 3(d) are obtained from

$$
\operatorname{Im}\left\{\chi_{y y z}^{(2)}\right\}=\left|\chi_{y y z}^{(2)}\right| \sin \left(\phi_{\mathrm{MEM}}+\phi_{\text {offset }}-\phi_{\mathrm{LLL}}\right) .
$$

We now return to the point that there are two values of $\phi_{\text {offset }}$ that satisfy the constraint $\operatorname{Im}\left\{\chi^{(2)}\right\} \rightarrow 0$ for $\omega_{\text {IR }}$ $<2800 \mathrm{~cm}^{-1}$ and $\omega_{\mathrm{IR}}>3200 \mathrm{~cm}^{-1}$. These differ by $\pm 180^{\circ}$ and result in opposite signs of $\operatorname{Im}\left\{\chi^{(2)}\right\}$ for the vibrational resonances. Since we do not have any explicit/experimental measurement of the $\chi^{(2)}$ phase, we could now make some chemical argument for the polarity of one of the molecules, tied to the sign of $\operatorname{Im}\left\{\chi^{(2)}\right\}$, and then make a proposal for a particular choice of $\phi_{\text {offset }}$. We choose not to do this and leave the ambiguity in the phase for future work. Even with this disclaimer, we still need to be careful that individual spectra within the heptane-toluene concentration series are consistent, that is, all with the correct phase, or all off by $180^{\circ}$. Note that this is not the same as providing a consistent choice of $\phi_{\text {offset }}$. We are confident that we have achieved this since many bands in Fig. 3(d) are monotonically increasing or decreasing. If the actual system displayed a flip in the polarity of heptane and/or toluene at a certain concentration, this would necessarily be accompanied by a decrease in mode amplitude (positive or negative) towards $\operatorname{Im}\left\{\chi^{(2)}\right\}=0$ followed by an increase in amplitude (negative or positive); this was not observed in our data. Finally, we note that this procedure cannot be applied to ppp spectra (shown in Fig. S1) since there are four contributing tensor elements: $\chi_{x x z}^{(2)}, \chi_{x z x}^{(2)}, \chi_{z x x}^{(2)}$, and $\chi_{z z z}^{(2)}$. Since we do not know the relative contribution of these elements (as they depend on the molecular orientation), we cannot apply the local field corrections. The ppp spectra are still valuable for confirming our mode assignments, but will not be considered further for our more detailed analysis.

\section{Spectral lineshape}

We are now in a position to take a closer look at the $\operatorname{Im}\left\{\chi_{y y z}^{(2)}\right\}$ spectra shown in Fig. 3(d). One observation is that the heptane $\mathrm{CH}_{3}$ symmetric stretch has an opposite sign to the toluene $\mathrm{CH}_{3}$ symmetric stretch, a clear indication that those two methyls have their $C_{3}$ axes pointing in opposite directions. ${ }^{51-54}$ Although heptane has a methyl group at each end of the chain, since it contains an odd number of carbon atoms, an all-trans configuration of the alkyl chain would not result in complete cancellation of the methyl SFG signals; some degree of conformational flexibility would further reduce the heptane methyl symmetric stretch intensity. However, since we observe both methyl groups to have roughly comparable signals, this is an indication that the relative orientations of the molecules also contribute. In brief, if heptane is oriented with its $\mathrm{CH}_{3}$ axis in the plane of the surface, only the $\mathrm{CH}_{3}$ antisymmetric $\left(\omega_{5}\right)$ and the methylene symmetric $\left(\omega_{1}\right)$ stretching will have appreciable intensity. Conversely, if the $\mathrm{CH}_{3}$ axis adopts a more upright geometry, the $\mathrm{CH}_{3}$ symmetric $\left(\omega_{2}\right)$ and the $\mathrm{CH}_{2}$ antisymmetric peaks $\left(\omega_{4}\right)$ are expected to be the most intense. However, in the $\operatorname{Im}\left\{\chi^{(2)}\right\}$ spectra of pure heptane, the $\mathrm{CH}_{3}$ symmetric, the $\mathrm{CH}_{2}$ antisymmetric, and the $\mathrm{CH}_{3}$ antisymmetric modes are present, with the $\mathrm{CH}_{2}$ antisymmetric being the strongest and the other two peaks of comparable amplitude. Therefore, it appears that the $\mathrm{CH}_{3}$ axis of heptane is oriented roughly perpendicular to the hydrophobic polymer surface. We note that it is customary to arrive at such conclusions, and provide more quantitative descriptions of the molecular orientation, by fitting the modes to resonance lineshapes and using the ratio of fit amplitudes. As an example, a previous study that has monitored the SFG response of heptane-toluene mixtures at the silica surface found that the $\mathrm{CH}_{3}$ axis of heptane was oriented approximately parallel to the charged hydrophilic surface. ${ }^{21}$ Here, our goal is to extract the maximum information from the spectra that is possible without any fitting, on account of this highly congested region of the mid-infrared with many heptane and toluene modes.

\section{Correlation analysis}

\section{Bulk homospectral correlation}

We have already discussed some interesting correlations between spectral features in the SFG signals originating from the fluoropolymer-liquid interface and those in the bulk spectra as revealed by the IR-Raman spectra. This can 
be continued in a more systematic fashion by computing the complex-valued correlation function

$$
\begin{aligned}
\mathrm{X}\left(\omega_{1}, \omega_{2}\right) & =\left\langle\tilde{s}_{1}\left(\omega_{1}, x\right) \cdot \tilde{s}_{2}\left(\omega_{2}, x^{\prime}\right)\right\rangle \\
& =\Phi\left(\omega_{1}, \omega_{2}\right)+i \Psi\left(\omega_{1}, \omega_{2}\right)
\end{aligned}
$$

where $\tilde{s}(\omega, x)$ are the IR-Raman and/or SFG spectral data at frequency $\omega$ and toluene mole fraction $x$. In the case where $s_{1}=s_{2}$ and we use solely the IR-Raman or the SFG data, this is referred to as homospectral correlation. ${ }^{55-57}$ When $s_{1}$ comes from IR-Raman data, and $s_{2}$ from SFG data, we compute the heterospectral correlation. ${ }^{58-61}$ The spectral quantities appearing in Eq. (9) are mean-centered about the $n$ values of $x$ at each frequency $\omega$

$$
\tilde{s}(\omega, x)=s(\omega, x)-\frac{1}{n} \sum_{i=1}^{n} s\left(\omega, x_{i}\right) .
$$

Practical details about the calculation of X $\left(\omega_{1}, \omega_{2}\right)$ including Fourier-transform based and algebraic methods may be found elsewhere. ${ }^{55}$ The real part of the correlation function in Eq. (9) $\Phi\left(\omega_{1}, \omega_{2}\right)$ is referred to as the synchronous component, and indicates which spectral features are changing together as the mole fraction of toluene increases in the bulk. The imaginary part $\Psi\left(\omega_{1}, \omega_{2}\right)$ is known as the asynchronous component, and is used to reveal which spectral correlations are shifted or lagging with respect to concentration.

As a first example, consider the IR-Raman homospectral correlation, with synchronous component shown in Fig. 5(a). The intensity along the diagonal indicates which spectral features are changing the most when the toluene mole fraction increases. The other striking feature in $\Phi\left(\omega_{1}, \omega_{2}\right)$ is the large blocks of positive (red) and negative (blue) cross peaks. A positive synchronous cross peak at $\left(\omega_{1}, \omega_{2}\right)$ indicates that the signals at $\omega_{1}$ and $\omega_{2}$ in the IR-Raman spectra are both increasing or both decreasing as the toluene concentration changes. Likewise, a negative cross peak indicates that the intensity at one frequency is increasing while the other displays a drop in signal. The distribution of positive and negative cross peaks is therefore as expected for this simple binary mixture, since the aliphatic $\mathrm{C}-\mathrm{H}$ stretching region below $3000 \mathrm{~cm}^{-1}$ contains contributions from both species, but is dominated by heptane. This region then decreases in intensity as $x$ increases and the aromatic modes grow. It is important to note that no component of the asynchronous map [Fig. 5(b)] is greater than $8 \%$ of the largest synchronous peak. In other words, all spectral changes are occurring synchronously in the bulk.
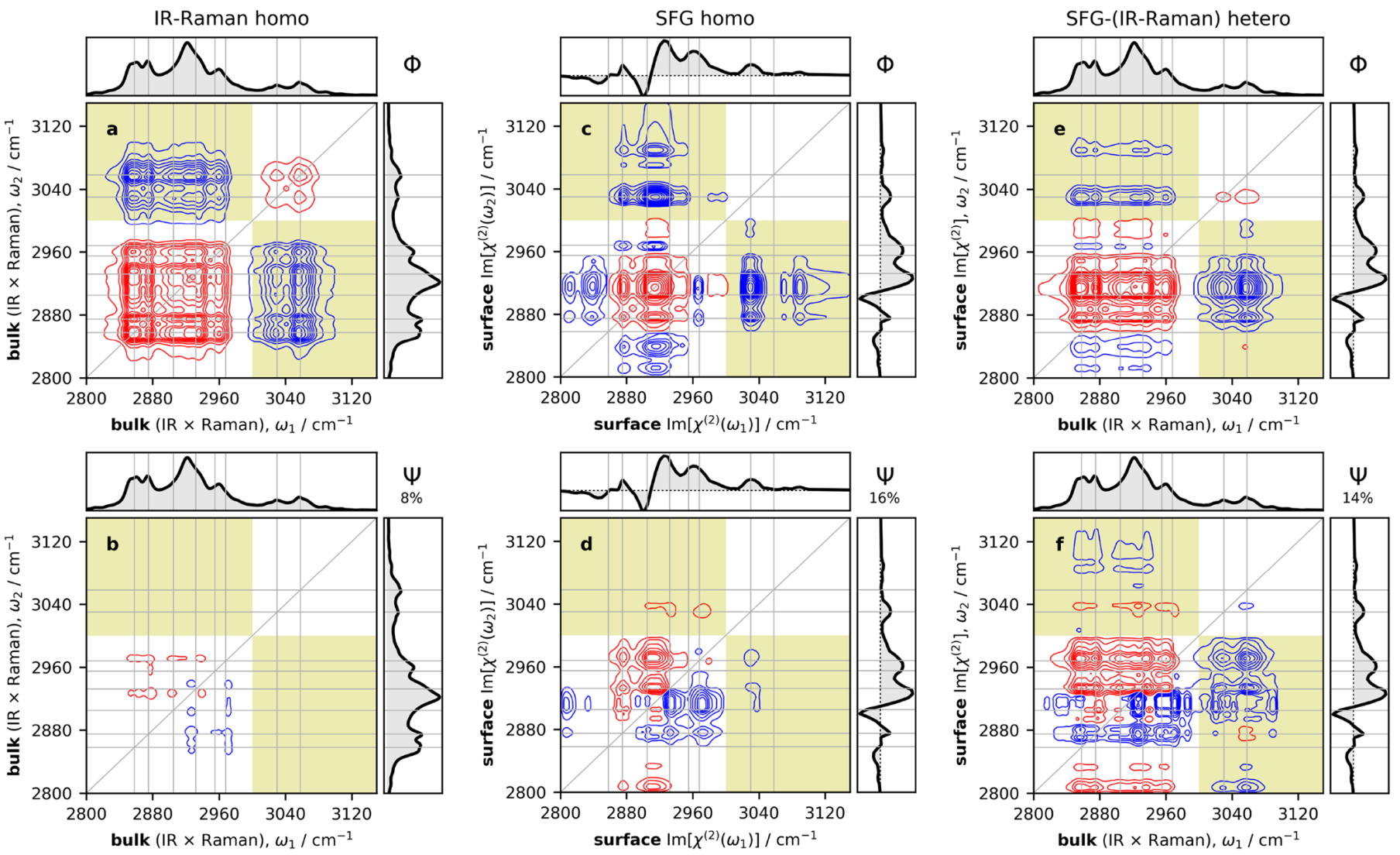

FIG. 5. Synchronous (top row) and asynchronous (bottom row) [(a) and (b)] homospectral IR-Raman, [(c) and (d)] homospectral SFG, and [(e) and (f)] heterospectral correlation maps. Regions about $3000 \mathrm{~cm}^{-1}$ are shaded yellow to highlight the predominantly aromatic stretching frequency range. 


\section{Surface homospectral correlation}

The homospectral correlation is generally more featurerich when applied to SFG data, as both the orientation and population of molecules at the surface contribute to the intensity of the vibrational modes. ${ }^{38,62-64}$ For example, a particular mode may have a strong IR transition dipole moment and Raman transition polarizability, but that part of the molecule may be oriented close to the plane of the surface, significantly reducing its intensity in the case of our $\mathrm{C}_{\infty v}$ symmetry. The orientation and symmetry consideration is the dominant contribution to the SFG, as even molecules with strong IR and Raman transitions are SFG-silent in the bulk solution phase. Again, this is the reason why composite IR-Raman spectra are useful to compare with SFG. The homospectral SFG correlation map is displayed in Fig. 5(c), with autopeaks dominated by $\omega_{2}, \omega_{3}$, and $\omega_{4}$. Looking at the cross peaks, we observe that all aliphatic modes decrease in amplitude with increasing bulk toluene concentration, except for the strong negative band at $\omega_{3}$ that grows (becomes more negative) with increasing toluene. Note that, even though the $\left(\omega_{3}, \omega_{6}\right)$, and $\left(\omega_{3}, \omega_{7}\right)$ cross peaks appear negative (blue color), they indicate a positive correlation since the band in $\operatorname{Im}\left\{\chi^{(2)}\right\}$ at $\omega_{3}$ is itself negative; that is, it is becoming increasingly negative. Similarly, the positive $\left(\omega_{2}, \omega_{3}\right)$ peak is indicative of a negative correlation. Now considering the asynchronous component in Fig. 5(d), the first thing to notice is that the largest component is only $16 \%$ of the largest synchronous peak. This is atypical of SFG homospectral correlation since the signal, in general, is susceptible to change when the surface species or their orientation/conformation change. The low asynchronous signals therefore indicate that there is only a small change in the heptane and toluene structure as the surface becomes more toluene-rich. The SFG homospectral asynchronous map has most cross peaks associated with either $\omega_{3}$ or $\omega_{6}$, that is, between heptane and toluene. For these species, we cannot distinguish reorientation from concentration lag. The possibility of a re-orientation of heptane and toluene can be deduced by examining peaks in the asynchronous maps that belong to only one molecule. For example, the strong negative correlation of the $\left(\omega_{3}, \omega_{6}\right)$ toluene peak reveals that the methyl antisymmetric mode changes before the methyl symmetric mode. This suggests that there is a re-orientation of the toluene methyl group with respect to the surface when there is heptane present in solution. For heptane, there is a weak negative correlation at the $\left(\omega_{2}, \omega_{4}\right)$ peak, which tells us that methylene antisymmetric mode changes before the methyl symmetric mode, suggesting a very slight re-orientation or conformation of heptane in the presence of toluene. We conclude that heptane reorientation is not significant. In other words, heptane structure at the surface is not affected much by the presence of toluene, but toluene orientation depends more significantly on how much heptane is there.

Yang et al. used their fitting of the $3022 \mathrm{~cm}^{-1}$ mode to create an absorption isotherm, ${ }^{21}$ shown with the red circles in Fig. 6. All of the points fell below the dashed equimolar line, indicating that the hydrophilic surface had a very slight preference for heptane. As our asynchronous $\operatorname{Im}\left\{\chi^{(2)}\right\}$ intensity is

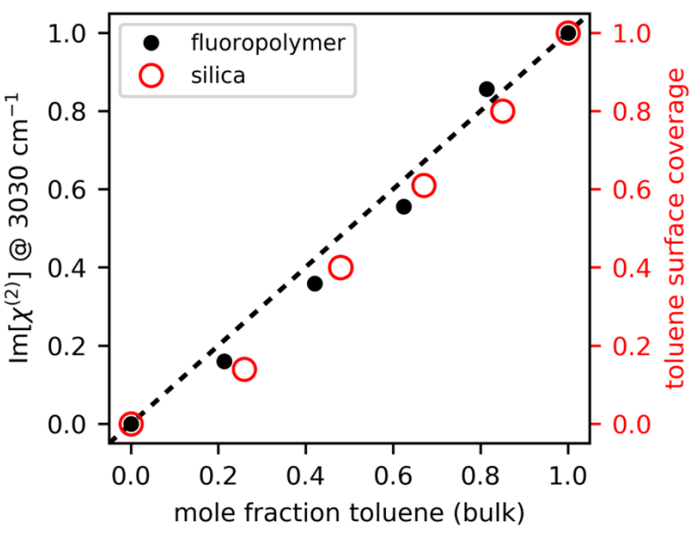

FIG. 6. Intensity of the $\operatorname{Im}\left\{\chi^{(2)}\right\}$ peak at $3030 \mathrm{~cm}^{-1}$ (black points) at the fluoropolymer surface as a function of the bulk mole fraction toluene, plotted together with the surface coverage data on silica from Ref. 21 (red circles).

not zero, we have to be cautious to interpret the results of a similar analysis; a plot of $\operatorname{Im}\left\{\chi^{(2)}\right\}$ at $3030 \mathrm{~cm}^{-1}$ is superimposed on Fig. 6 with black points. Considering that there is indication of structural variation in our homospectral SFG data, there is likely no significant preference for heptane over toluene on the hydrophobic surface.

\section{Surface-bulk heterospectral correlation}

In order to interpret the surface-bulk heterospectral correlation results, there are three limiting cases to consider: (1) The homospectral $\operatorname{Im}\left\{\chi^{(2)}\right\}$ asynchronous signal is large, in which case it becomes challenging to interpret the heterospectral correlation. (2) The homospectral $\operatorname{Im}\left\{\chi^{(2)}\right\}$ asynchronous signal is negligible, and the heterospectral surfacebulk asynchronous component is large. Here, we know that the surface spectral changes are entirely due to changes in surface population (i.e. the adsorbed orientation of heptane and toluene are not concentration-dependant), and we can tell the sequence of the surface vs bulk population change, revealing whether the surface favours heptane or toluene. Note that heterospectral asynchronous correlations between the same IR frequency at the surface and in the bulk cannot be used for this purpose, as the corresponding homospectral $\operatorname{Im}\left\{\chi^{(2)}\right\}$ peaks are absent; that is, there are never homospectral asynchronous diagonal peaks. (3) The homospectral $\operatorname{Im}\left\{\chi^{(2)}\right\}$ asynchronous and heterospectral surface-bulk asynchronous signals are both negligible. In this situation, we know that the surface structure is not concentration-dependent and the surface has no preference for either molecule.

The surface-bulk heterospectral asynchronous correlation map appears in Fig. 5(f). Following the above guidelines, we select only those cross peaks that are very weak or absent in homospectral asynchronous correlation [Fig. 5(d)] for further analysis. The negative peak at $\left(\omega_{8}, \omega_{7}\right)$ is a toluene mode that is positive in the corresponding heterospectral synchronous map [Fig. 5(e)]. This could suggest that, as the bulk concentration of toluene increases, spectral changes occur at the surface before the bulk. However, examining the peaks 
$\left(\omega_{1}, \omega_{2}\right),\left(\omega_{1}, \omega_{4}\right),\left(\omega_{5}, \omega_{2}\right)$, and $\left(\omega_{5}, \omega_{4}\right)$ originating from heptane modes reveals a different trend. Peaks $\left(\omega_{1}, \omega_{2}\right)$ and $\left(\omega_{5}, \omega_{2}\right)$ are negative in the asynchronous map and positive in the corresponding synchronous map, which informs us that as the bulk concentration of toluene increases spectral changes occur at the surface before the bulk. However, cross peaks $\left(\omega_{1}, \omega_{4}\right)$ and $\left(\omega_{5}, \omega_{4}\right)$ are positive in the asynchronous and synchronous maps, which informs us that as the bulk concentration of toluene increases spectral changes occur in the bulk solution before they do at the surface. Therefore, this indicates that the polymer surface does not show a significant preference for either toluene or heptane, very similar to what we gleaned from the plot of the $\operatorname{Im}\left\{\chi^{(2)}\right\}$ signal at $3030 \mathrm{~cm}^{-1}$ with respect to the bulk toluene mole fraction (Fig. 6).

It is worth noting that the negative asynchronous peaks (and positive in synchronous) both include the $\mathrm{CH}_{3}$ symmetric stretch of heptane $\left(\omega_{2}\right)$, and both positive synchronous and asynchronous peaks include the $\mathrm{CH}_{2}$ asymmetric stretch of heptane $\left(\omega_{4}\right)$. Future work is aimed at understanding such relationships in the context of the molecular orientation and chain conformation, extending our efforts to gain structural insight in cases where spectra are too congested to fit, and multiple polarization schemes are not accessible or cannot be treated quantitatively.

\section{SUMMARY}

Traditionally, SFG spectra are fit using a resonance lineshape such as a sum of Lorentzians, and the change in orientation may be determined from the ratio of peak amplitudes measured in two or more polarization schemes. A challenge arises when the spectral region is sufficiently congested so as to make spectral fitting difficult or impossible. In such cases, a combination of SFG homospectral and surface-bulk heterospectral 2DCOS is a valuable alternative. The rules for interpreting SFG homospectral and heterospectral correlation maps presented above are strictly true only when using $\operatorname{Im}\left\{\chi^{(2)}\right\}$ data obtained from the SFG spectra. If $\left|\chi^{(2)}\right|^{2}$ spectra were used in the 2DCOS, artifacts from the changing interference between neighbouring modes would obscure these trends. ${ }^{62,63}$ We have used these general principles to determine that there is only a small change in orientation of toluene as their surface composition changes, and no obvious preference for either molecule on a perfluorinated hydrophobic surface. These results, together with previous understanding of the structure at hydrophilic surfaces, provide a foundation for understanding adsorption of real and model constituents such as asphthalenes, and the further optimization of anti-fouling coatings for alkane- and aromatic-based fluids.

\section{SUPPLEMENTARY MATERIAL}

See supplementary material Fig. S1 for uncorrected IR reflection absorbance, Raman scattering, and SFG $\left|\chi_{\text {effective }}^{(2)}\right|^{2}$ spectra in ssp and ppp polarizations for pure heptane toluene.

\section{ACKNOWLEDGMENTS}

We thank the Natural Sciences and Engineering Research Council of Canada (NSERC) for support of this science with a Discovery Grant and Imperial Oil for a University Research Award. NSERC in partnership with Imperial Oil provided funding with a Collaborative Research and Development grant. Tasha Jarisz provided experimental assistance, and Sandra Roy participated in the discussion of the 2DCOS results. Stanislav Konorov assisted in the collection of the Raman spectra at the University of Victoria CAMTEC facility.

\section{REFERENCES}

${ }^{1}$ T. R. Bott, Fouling of Heat Exchangers (Elsevier Science and Technology Books, Amsterdam, 1995).

${ }^{2}$ Z. Fan, P. Rahimi, T. Alem, A. Eisenhawer, and P. Arboleda, Energy Fuels 25, 1182 (2011)

${ }^{3}$ A. P. Watkinson, B. Navaneetha-Sundaram, and D. Posarac, Energy Fuels 14, 64 (2000).

${ }^{4}$ S. Asomaning and A. P. Watkinson, Can. J. Chem. Eng. 70, 444 (1992).

${ }^{5}$ D. A. Bartlett, Indust. Phys. 2(4), 18 (1996).

${ }^{6} \mathrm{~K}$. Brodowicz and M. Markowski, Appl. Thermal Eng. 23, 2241 (2003).

${ }^{7}$ H. Belhaj, H. A. Khalifeh, and N. Al-Huraibi, Petrol. Environ. Biotech. 4, 142 (2013).

${ }^{8}$ C. A. Bennett, Heat Transfer Eng. 33, 1246 (2012).

${ }^{9}$ S. Maccietto, G. F. Hewitt, F. Coletti, B. D. Crittenden, D. R. Dugwell, A. Galindo, G. Jackson, R. Kandiyoti, S. G. Kazarian, P. F. Luckham, O. K. Matar, M. Millan-Agorio, E. A. Muller, W. Paterson, S. J. Pugh, S. M. Richardson, and D. I. Wilson, Heat Transf. Eng. 32, 197 (2011).

${ }^{10}$ A. P. Watkinson, Heat Transfer Eng. 28, 177 (2007).

${ }^{11}$ J. D. McLean and P. K. Kilpatrick, J. Colloid Interface Sci. 196, 23 (1997).

${ }^{12}$ V. Venditti, C. Berrueco, P. Alvarez, T. J. Morgan, M. Millan, A. A. Herod, and R. Kandiyoti, Proc. Int. Conf. Heat Exch. Fouling Clean. 8, 86 (2009).

${ }^{13}$ S. Asomaning and A. P. Watkinson, Heat Transfer Eng. 21, 10 (2000).

${ }^{14}$ I. A. Wiehe, R. J. Kennedy, and G. Dickakian, Energy Fuels 15, 1057 (2001).

${ }^{15}$ K.-H. Kim, D.-H. Yeu, S. Ha, and S.-K. Koh, Heat Exch. Foul. Clean. 13, 91-99 (2004).

${ }^{16}$ D. J. Kukulka and P. Leising, Chem. Eng. Trans. 18, 785 (2009).

${ }^{17} \mathrm{~W}$. Wang, P. Dowson, and A. Baha, in Proceedings of the Thirty-Second Turbomachinery Symposium (Texas A\&M University Turbomachinery Laboratories, 2003), pp. 91-97.

${ }^{18}$ R. W. Boyd, Nonlinear Optics, 2nd ed. (Academic Press, San Diego, 2003).

${ }^{19}$ Y. R. Shen, The Principles of Nonlinear Optics (John Wiley \& Sons, New York, 1984).

${ }^{20}$ A. Morita, Theory of Sum Frequency Generation Spectroscopy (Springer, Singapore, 2018).

${ }^{21}$ Z. Yang, Q. Li, R. Hua, M. R. Gray, and K. C. Chou, J. Phys. Chem. C 113, 20355 (2009).

${ }^{22}$ W.-C. Yang and D. K. Hore, J. Chem. Phys. 149, 174703 (2018).

${ }^{23}$ B. Busson and A. Tadjeddine, J. Phys. Chem. C 113, 21895 (2009).

${ }^{24}$ K. C. Jena and D. K. Hore, J. Phys. Chem. C 113, 15364 (2009).

${ }^{25}$ J. K. Wilmshurst and H. J. Bernstein, Can. J. Chem. 35, 911 (1957).

${ }^{26}$ F. F. Cleveland and P. Porcelli, J. Chem. Phys. 18, 1459 (1950).

27J. L. Achtyl, A. M. Buchbinder, and F. M. Geiger, J. Phys. Chem. Lett. 3, 280 (2012).

${ }^{28}$ E. L. Hommel and H. C. Allen, Analyst 128, 750 (2003).

${ }^{29}$ C. S.-C. Yang, P. T. Wilson, and L. J. Richter, Macromolecules 37, 7742 (2004)

${ }^{30}$ H. R. Phillip and E. A. Taft, Phys. Rev. 136, A1445 (1964).

${ }^{31}$ Y. Shindo and K. Kusano, J. Chem. Eng. Data 24, 106 (1979).

${ }^{32}$ G. Baranović, Appl. Spectrosc. 71, 1039 (2017). 
${ }^{33}$ M. R. Anderson, "Determination of infrared optical constants for single component hydrocarbon fuels," M.S. thesis, University of Missouri-Rolla, Rolla, MO, 2000.

${ }^{34} \mathrm{M}$. Milosevic, Internal Reflection and ATR Spectroscopy (John Wiley \& Sons, Inc., Hoboken, NJ, 2012).

${ }^{35}$ N. J. Harrick, Internal Reflection Spectroscopy (Interscience Publisher, John Wiley \& Sons, New York, NY, 1967).

${ }^{36}$ E. O. Potma and S. Mukamel, in Coherent Raman Scattering Microscopy (CRC Press, Boca Raton, 2013), Chap. 1, pp. 3-42.

${ }^{37}$ R. L. McCreery, Raman Spectroscopy for Chemical Analysis (WileyInterscience, New York, 2000).

${ }^{38}$ S. Roy, C. Beutier, and D. Hore, J. Mol. Struct. 1161, 403 (2018).

${ }^{39}$ P. H. Axelsen and M. J. Citra, Prog. Biophys. Mol. Biol. 66, 227 (1996).

${ }^{40}$ W. N. Hansen, J. Opt. Soc. Am. 58, 380 (1968).

${ }^{41}$ F. Abelès, Ann. Phys. 12, 596 (1950).

${ }^{42}$ D. B. O'Brien and A. M. Massari, J. Opt. Soc. Am. B 30, 1503 (2013).

${ }^{43}$ D. O'Brien and A. Massari, J. Chem. Phys. 138, 154708 (2013).

${ }^{44}$ W. R. FitzGerald, K. C. Jena, and D. K. Hore, J. Mol. Struct. 1084, 368 (2014).

${ }^{45}$ A. G. F. de Beer, J.-S. Samson, W. Hua, Z. Huang, X. Chen, H. C. Allen, and S. Roke, J. Chem. Phys. 135, 224701 (2011).

${ }^{46}$ M. J. Hofmann and P. Koelsch, J. Chem. Phys. 143, 134112 (2015).

${ }^{47}$ P. K. Johansson and P. Koelsch, J. Am. Chem. Soc. 136, 13598 (2014).

${ }^{48}$ M. Sovago, E. Vartiainen, and M. Bonn, J. Phys. Chem. C 113, 6100 (2009).

${ }^{49}$ P.-K. Yang and J. Y. Huang, J. Opt. Soc. Am. B 14, 2443 (1997).
${ }^{50}$ P.-K. Yang and Y. Y. Huang, J. Opt. Soc. Am. B 17, 1216 (2000).

${ }^{51}$ S. Nihonyanagi, S. Yamaguchi, and T. Tahara, J. Chem. Phys. 130, 204704 (2009).

${ }^{52}$ K. C. Jena, P. A. Covert, S. A. Hall, and D. K. Hore, J. Phys. Chem. C 115, 15570 (2011)

${ }^{53}$ W.-C. Yang and D. K. Hore, J. Phys. Chem. C 121, 28043 (2017).

${ }^{54}$ W.-C. Yang and D. K. Hore, J. Phys. Chem. C 122, 18192 (2018).

${ }^{55}$ I. Noda and Y. Ozaki, Two-Dimensional Correlation Spectroscopy: Applications in Vibrational and Optical Spectroscopy (John Wiley \& Sons, Ltd., San Francisco, 2004).

${ }^{56}$ I. Noda, Bull. Am. Phys. Soc. 31, 520 (1986).

${ }^{57}$ I. Noda, J. Am. Chem. Soc. 111, 8116 (1989).

${ }^{58}$ I. Noda, Y. Liu, and Y. Ozaki, J. Phys. Chem. 100, 8674 (1996).

${ }^{59}$ H. J. Kim, S. B. Kim, J. K. Kim, and Y. M. Jung, J. Phys. Chem. 110, 23123 (2006).

${ }^{60}$ T. Genkawa, M. Watari, T. Nishii, M. Suzuki, and Y. Ozaki, Appl. Spectrosc. 67, 724 (2013).

${ }^{61}$ C. Zou, M. Larisika, G. Nagy, J. Srajer, C. Oostenbrink, X. Chen, W. Knoll, B. Liedberg, and C. Nowak, J. Phys. Chem. B 117, 9606 (2013).

${ }^{62}$ S. Roy, J. S. Post, K.-K. Hung, U. Stege, and D. K. Hore, J. Mol. Struct. 1069, 103 (2014).

${ }^{63}$ S. Roy, P. A. Covert, T. A. Jarisz, C. Chan, and D. K. Hore, Anal. Chem. 88, 4682 (2016).

${ }^{64}$ S. Roy, S. Freiberg, C. Leblanc, and D. K. Hore, Langmuir 33, 1763 (2017). 